Louisiana State University

LSU Digital Commons

$5-9-2019$

\title{
Molecularly Heterogeneous Structure of a Nonionic Deep Eutectic Solvent Composed of N-Methylacetamide and Lauric Acid
}

\author{
Yaowen Cui \\ Louisiana State University \\ Jeramie C. Rushing \\ Louisiana State University \\ Soenke Seifert \\ Argonne National Laboratory \\ Nicholas M. Bedford \\ UNSW Sydney \\ Daniel G. Kuroda \\ Louisiana State University
}

Follow this and additional works at: https://digitalcommons.Isu.edu/chemistry_pubs

\section{Recommended Citation}

Cui, Y., Rushing, J., Seifert, S., Bedford, N., \& Kuroda, D. (2019). Molecularly Heterogeneous Structure of a Nonionic Deep Eutectic Solvent Composed of N-Methylacetamide and Lauric Acid. Journal of Physical Chemistry B, 123 (18), 3984-3993. https://doi.org/10.1021/acs.jpcb.8b11732

This Article is brought to you for free and open access by the Department of Chemistry at LSU Digital Commons. It has been accepted for inclusion in Faculty Publications by an authorized administrator of LSU Digital Commons.

For more information, please contact ir@lsu.edu. 


\section{The Molecularly Heterogeneous Structure of Non-Ionic Deep Eutectic Solvent Composed of N-Methylacetamide and Lauric Acid}

Yaowen Cui,† Jeramie Rushing, † Soenke Seifert $¥$ \$ Nicholas M. Bedford,§ and Daniel G. Kuroda†*

†Department of Chemistry, Louisiana State University, Baton Rouge, Louisiana 70803, United

States

$\ddagger X$-ray Sciences Division, Argonne National Laboratory, Argonne, IL, 60439, USA

§School of Chemical Engineering, University of New South Wales, Sydney, NSW, 2052, AUS

*Address correspondence to dkuroda@lsu.edu 


\begin{abstract}
Deep eutectic solvents (DES) are a new class of designer solvents with a wide range of possible applications. DESs are typically made from an ionic compound and a hydrogen bond donor, but lately DESs made of non-ionic compounds with interesting properties have started to appear. This study focuses on describing the structure, dynamics, and interactions of a non-ionic DES composed of N-methylacetamide and lauric acid. Linear IR and time-resolved IR experiments show that LANMA DES is the result of hydrogen bond interaction between the two molecular components, though the interaction energy is only favor by $\sim 1 \mathrm{~kJ} / \mathrm{mol}$ with respect to the pure components. The IR experiments also reveal the presence of molecular heterogeneities produced by the nanosegregation of polar and non-polar domains formed by N-methylacetamide and lauric acid, respectively. In addition, it is observed that the hydrogen bond interaction between components occurs at the interface of the two distinct polar domains. The proposed molecular picture is also supported by the observation of nano-domains with the approximate size of two lauric acids via small angle X-ray scattering.
\end{abstract}




\section{INTRODUCTION}

Deep Eutectic Solvents (DESs) are liquids formed by mixing two solids with specific chemical structures. ${ }^{1}$ Based on the eutectic phenomenon, the fusion point of the DES is lower than the melting point of its individual components. ${ }^{1}$ The use of solid components to prepare the DESs results in liquids with negligible vapor pressure. ${ }^{2-3}$ Moreover, due to their easy preparation, the physical and/or chemical properties of DESs can be tailored to specific applications by selecting specific pairs of components. ${ }^{4-7}$ Because of these properties, DESs are considered as designer solvents. $^{8}$

DESs have some advantages compared to classical designer solvents, such as ionic liquids. The materials for preparing DES can be specifically selected to be inexpensive, safe, and easy to produce. ${ }^{5-6}$ In addition, the simple DES synthesis, consisting only on mixing, lowers significantly the preparation cost, allowing the use of DESs in a wide range of fields. ${ }^{6,8-9}$ Finally, DES properties are tailorable not only by varying the component chemical identity, like ionic liquids, but also the molar ratio of components. ${ }^{5,10}$ Due to these advantages, DESs have been extensively studied and applied in a wide range of scientific and engineering areas, such as: organic synthesis, ${ }^{6,} 11$ electrochemistry, ${ }^{12-13}$ metal oxide dissolution, ${ }^{14}$ carbon dioxide absorption, ${ }^{15}$ separation processes, ${ }^{16}$ polymerizations, ${ }^{17-18}$ and lately in oil-spill remediation. ${ }^{19}$

The most studied DESs are those composed of quaternary ammonium chloride or metal chloride (hydrate), which are the so-called “ionic” DESs. ${ }^{2}$ In particular, DESs based on choline salts have been the most investigated in recent years. Moreover, the dynamics and structure of choline-based DESs have been extensively studied with either experimental or theoretical methods. ${ }^{20-25}$ Based on the ionic nature of the DES components, it is not surprising that the resulting ionic DESs are hydrophilic and have polarities larger than ionic liquids. ${ }^{26}$ However, there 
is a large demand for low polarity DESs in applications such as organic chemistry and extraction of natural products. ${ }^{27-28}$ Hydrophobic DESs have been reported in the literature as extractants which are immiscible water. ${ }^{28}$ In particular these hydrophobic DESs are composed of a long chain carboxylic acid and various quaternary ammonium salts, where the latter component has three or four carbon alkyl chains. Although these hydrophobic DESs have ionic components, they present low solubility of water as observed by the low amount of quaternary ammonium salt leaching into water when the DES is in contact with it. The low leaching of the ionic component into water demonstrates a strong interaction between the chloride ion and the acid, which is similar to cholinebased DESs. Other non-ionic hydrophobic DESs have been previously studied. For example, acetamide-urea mixtures have been shown to form non-ionic DESs with both structural and dynamical homogeneity. ${ }^{29}$ In this case the structural homogeneity is achieved by the chemical similarity of the DES components (acetamide and urea), and the DES behaves more like an ideal solution than a DES since it is likely that the driving force for their formation is the entropy of mixing. Our group has previously reported a DES composed of N-methylacetamide (NMA, Scheme 1) and lauric acid (LA, Scheme 1), which is hydrophobic and non-ionic. ${ }^{5,19}$ In this DES, the mixtures contain two different species that strongly differ in molecular size, water solubility, and polarity. The disparity in the chemical properties of the NMA-LA DES components is manifested by the addition of water, which produces the precipitation of the hydrophobic component (LA) of the DES. ${ }^{19}$ Thus, the NMA-LA DES is expected to have a more heterogeneous molecular structure than that reported for acetamide/urea. ${ }^{29}$ Moreover, the separation of the DES components in the presence of water is likely the result of relatively weak interactions between the NMA and LA molecules, as well as the dissimilarity in the chemical structure (polarity) of the two components. Therefore, the much weaker interaction and dissimilar chemical structure between 
the non-ionic DES components is expected to play a significant role in defining the molecular structure and interactions in the DES. However, the investigation of the interaction between components in the NMA-LA non-ionic DES is yet to be addressed.

(a)

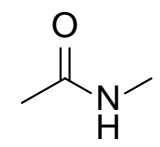

(b)

$\mathrm{H}$

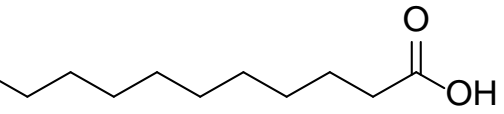

Scheme 1. Structure of (a) N-methylacetamide (NMA) and (b) lauric acid (LA).

This work is dedicated to investigating the structure and interaction between components in the NMA-LA DES and the molar ratio effect on the structure of the resulting liquid. In particular, the study is focused on the DES composed of LA:NMA at molar ratios of 1:2, 1:4, and 1:6, where the ratio of 1:4 is very close to the eutectic point. Note that the fusion point of the mixtures of LA:NMA at the molar ratios from 1:2 to 1:6 is below room temperature, which provides us with a big window to study the interaction between components with different molar fractions. ${ }^{19}$ For this purpose, the amide I vibrational mode of NMA as well as the carbonyl stretch of LA are employed as probes to report the interaction with the environment. These two vibrational modes are used because of their sensitivity to the molecular environment, ${ }^{30-32}$ which directly affects the vibrational mode as observed by the changes in its vibrational dynamics and resonance frequency. ${ }^{33-34}$ For example, one can easily observe the number of hydrogen bonds formed by either amide or carboxylic acids due to the distinct resonant frequency of the amide/carbonyl stretch as a function of the number of hydrogen bonds. ${ }^{33-38}$ Thus, Fourier Transform Infrared (FTIR) and twodimensional infrared spectroscopy (2DIR) are used to study the vibrational dynamics of each probe, from which one can decode the structure and interactions of each component in the mixture. Moreover, photon echo spectroscopy provides information about processes, such as chemical 
exchange and spectral diffusion, which are important to obtaining a molecular picture of the interactions and motions occurring thermally in the sample at sub- or picosecond timescale. ${ }^{39-40}$

\section{EXPERIMENTAL METHODOLOGIES}

\section{Sample preparation}

Lauric acid (Alfa Aesar, 99.5\%) and N-methylacetamide (Alfa Aesar, 99\%) were used as received for preparing the samples. The two components were mixed at varied ratios. The samples were prepared by mixing the two components in a vial with either a vortex mixer or sonicator until a liquid was formed. Note that heating was not required for preparing the DES. All the samples were stored and prepared in a nitrogen-flushed glovebox.

\section{Linear IR spectroscopy}

Linear IR measurements were performed using a Bruker Tensor 27 with a liquid nitrogen cooled narrow band MCT detector. All of the samples were measured with $0.5 \mathrm{~cm}^{-1}$ resolution, and all the spectra were averaged from 40 scans at room temperature. The samples were held in an o-ring sealed sample cell with two $\mathrm{CaF}_{2}$ windows and separated by Teflon spacers. All the sample cells were prepared in a nitrogen-filled glovebox to minimize exposure to moisture.

\section{Two-dimensional IR spectroscopy}

The 2DIR spectroscopy measurements were performed using a similar setup and procedures previously described in the. ${ }^{36}$ In short, the broadband infrared pulse was generated from a SpectraPhysics Spitfire Ace Ti: Sapphire amplifier with a $5 \mathrm{kHz}$ repetition rate and an optical parametric amplifier (Spectra-Physics OPA-800C). The photon echo of the different samples was generated by overlapping three replica IR pulses, with wave vectors of $\boldsymbol{k}_{1}, \boldsymbol{k}_{2}$, and $\boldsymbol{k}_{3}$ respectively, in the sample in a boxcar configuration. ${ }^{39}$ The photon echo at the phase matching the direction $-\boldsymbol{k}_{1}+\boldsymbol{k}_{2}$ $+\boldsymbol{k}_{3}$ was overlapped with a fourth pulse (local oscillator) to achieve heterodyne detection. The 
interference of the photon echo and local oscillator was detected by a liquid nitrogen cooled MCT 64 element array detector with a monochromator. The time intervals between pulses $\tau$ (between first and second pulses), $\mathrm{T}_{\mathrm{w}}$ (between second and third pulses), and $\mathrm{t}$ (between third and the photon echo) were controlled by translation stages. For the experimental measurements, the $\tau$ was scanned from -4 ps to 4 ps with a step 5 fs for each waiting time, and the local oscillator always preceded the signal by $\sim 0.5$ ps. To analyze the data, the signal in the time domain $\left(S\left[\tau, T_{w}, t\right]\right)$, was transformed to the signal in the frequency domain by double Fourier Transform of the two coherence times ( $\tau$ and $t$ ) for the different waiting times $\left(T_{W}\right) .{ }^{41}$ For the 2DIR measurements of the pure DES, no spacer was used. A lens sample cell was used to reduce the absorbance when acquiring the 2DIR signal of amide I band in DES, using the same procedure as previously reported. ${ }^{42}$

\section{Small angle \& wide-angle $X$-ray scattering}

Small angle X-ray scattering (SAXS) and wide-angle X-ray scattering measurements were performed at the 12-ID-B station of the Advanced Photon Source (APS), Argonne National Laboratory (ANL). Samples were loaded into thin-walled quartz X-ray capillaries for all measurements using $14 \mathrm{keV}$ X-rays, $1.0 \mathrm{~s}$ exposure time, and a sample to SAXS detector distance of $1997.08 \mathrm{~mm}$. SAXS data was collected on a Pilatus 2M detector while WAXS data was collected on a Pilatus 300k detector. Data reduction and background correction was performed onsite for further analysis.

\section{Differential scanning calorimetry (DSC)}

The DSC measurements were performed on a DSC 2920 (TA instruments) using an empty pan as the reference. The sample $(\sim 1 \mathrm{~mL})$ and the reference pan were held on a hermetic alodined pan (TA instruments). The DSC cycle consisted of first cooling the LA:NMA mixture and the 
reference pan to $-40{ }^{\circ} \mathrm{C}$, where they remained for $30 \mathrm{~min}$ to achieve equilibrium, followed by a heating step with a rate of $10^{\circ} \mathrm{C}$ per min to achieve a final temperature of $80^{\circ} \mathrm{C}$.

\section{RESULTS}

The phase diagram of the mixture of NMA and LA was first examined via DSC to assess the composition of the eutectic point. From the DSC thermograms, all the transition temperatures and those of the pure LA and NMA were obtained (Figure 1). In the mixtures with a LA:NMA molar ratios of 1:4 and 1:5 only one endothermic peak was observed at $\sim 6^{\circ} \mathrm{C}$, while other mixtures showed two endotherms in the thermogram. All the mixtures presented a similar low transition temperature of $\sim 7^{\circ} \mathrm{C}$, which indicated the presence of a eutectic at a composition of LA:NMA molar ratio of 1:4-5.

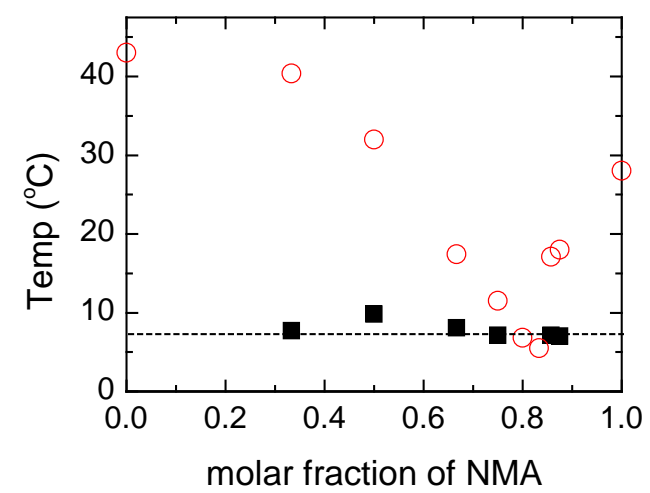

Figure 1. Phase transition temperatures of the mixture of NMA-LA determined by DSC. Red circles and black squares represent the first and second transition temperatures of the mixture. Dashed lines are added as a guide to the eye.

The study of the structure of the NMA-LA DES was investigated via linear IR spectroscopy. The normalized FTIR spectra of NMA-LA DES with varied molar ratios of NMA and LA (Figure 1) shows two groups of peaks corresponding to the vibrational transitions of NMA and LA. According to the assignment done previously, the former bands correspond to the amide I and II transition of NMA located in the frequency range $1600 \mathrm{~cm}^{-1}$ to $1700 \mathrm{~cm}^{-1}$, ${ }^{19}$ while the 
latter relate to the carbonyl bands of LA found in the frequency range $1700 \mathrm{~cm}^{-1}$ to $1775 \mathrm{~cm}^{-1}$. $^{20}$, ${ }^{34}$ In particular, the bands centered at $1566 \mathrm{~cm}^{-1}$ and $1656 \mathrm{~cm}^{-1}$ in the spectra correspond to the amide II and I bands of NMA, respectively. ${ }^{43}$ The amide I band of NMA in the different molar ratio mixtures presents an asymmetric profile with shoulders on both sides of the main peak located at $\sim 1630 \mathrm{~cm}^{-1}$ and $1685 \mathrm{~cm}^{-1}$. With increasing concentration of NMA (1:2 to 1:6 mixture), the ratio of area red-side band $\left(\sim 1630 \mathrm{~cm}^{-1}\right)$ to the center band increases. In contrast, it appears that the blue-side shoulder $\left(\sim 1685 \mathrm{~cm}^{-1}\right)$ does not show a significant change for the different mixtures.

Similar to the NMA bands, LA in the different mixtures presents two bands (Figure 1), which are at $\sim 1710 \mathrm{~cm}^{-1}$ and $\sim 1730 \mathrm{~cm}^{-1}$. The total absorbance of acid bands shows the expected decrease with the decreasing concentration of LA from 1:2 to 1:6 LA:NMA mixture, but the absorbance of the low-frequency band decreases more than the other acid band. The result indicates a change in ratio of areas of the two acid bands with concentration of LA.

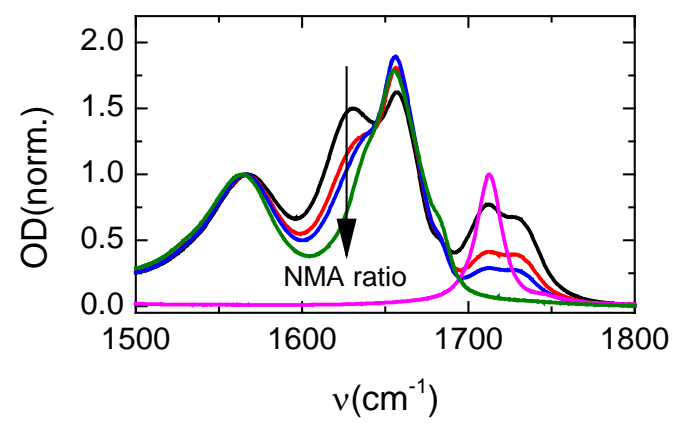

Figure 1. (a)Normalized FTIR spectra of acid carbonyl band of NMA-LA with a molar ratio of 2:1 (black line), 4:1 (red line), and 6:1 (blue line), pure NMA (green line), and LA (pink line).

The temperature dependent FTIR spectra were also measured for the mixtures with different molar ratios of NMA and LA. The FTIR spectra of LA-NMA mixtures at the temperatures from $10^{\circ} \mathrm{C}$ to $50^{\circ} \mathrm{C}$ every $10{ }^{\circ} \mathrm{C}$ are displayed in Figure 2. The temperature-dependence spectra shows a general behaviour among the different solutions: an increase (decrease) of the high (low) frequency band in both the NMA amide I and the LA carbonyl bands. In other words, as the 
temperature increases, the band centered at $\sim 1630 \mathrm{~cm}^{-1}$ decreases, and the band at $\sim 1656 \mathrm{~cm}^{-1}$ increases. While the effect is less noticeable for the two bands of LA, the spectra (Figure 2) shows that all the LA-NMA mixtures have a decrease (increase) in the intensity of the $\sim 1710 \mathrm{~cm}^{-1}(\sim 1730$ $\mathrm{cm}^{-1}$ ) bands with temperature.

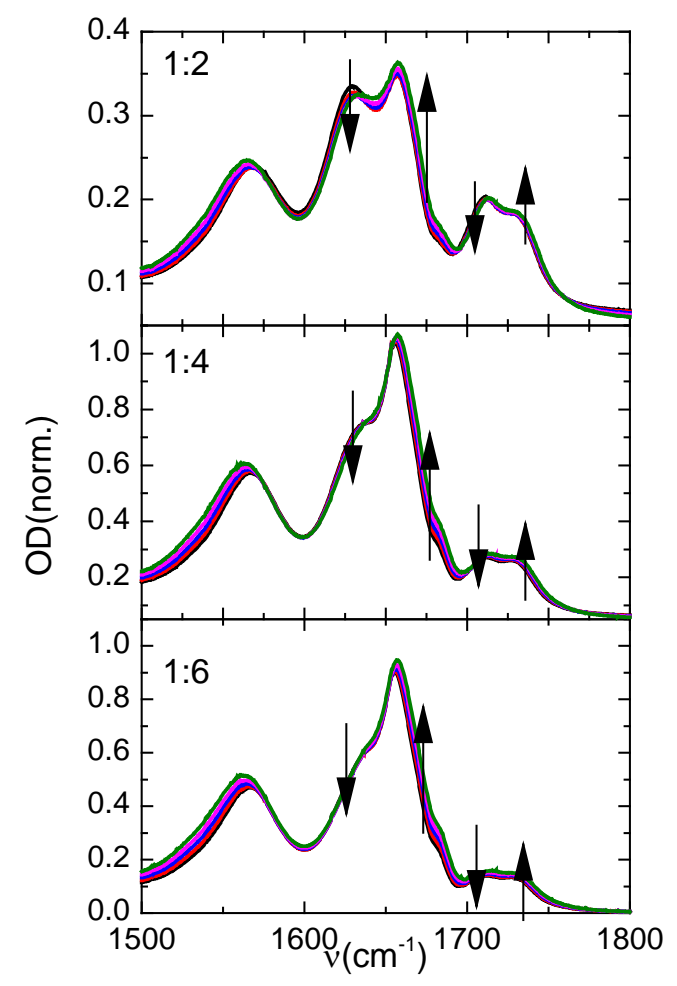

Figure 2. FTIR spectra of different LA:NMA mixtures with different temperatures at the amide I and carbonyl stretching range of NMA and LA. Arrows denote the change when temperature increases.

The structure and interactions of the different components in the NMA-LA mixtures were further studied by 2DIR spectroscopy. The 2DIR spectra centered at the amide band region for the different NMA-LA samples were measured (Figure 3). In the 2DIR spectra of Figure 3, the red peaks (positive) at high probe frequency along the diagonal line indicate vibrational transitions from $v=1$ to $v=0$. In contrast, the blue peaks (negative) refer to the transitions from $v=1$ to $v=$ 2. There are six pairs of peaks located along the diagonal line at short waiting time. The two pairs of peaks at $\sim 1710 \mathrm{~cm}^{-1}$ and $\sim 1730 \mathrm{~cm}^{-1}$ indicate the carbonyl stretching modes of LA with direct 
correspondence to those seen in the FTIR spectra. Moreover, the 2DIR spectra is very similar to the previously published 2DIR spectra of pure NMA. The other four pairs of peaks correspond to the amide vibrational modes of the NMA molecule. The peaks at $\sim 1556 \mathrm{~cm}^{-1}$ are amide II bands, and the three peaks at $\sim 1615 \mathrm{~cm}^{-1}, \sim 1649 \mathrm{~cm}^{-1}$, and $\sim 1674 \mathrm{~cm}^{-1}$ are amide I bands of NMA. ${ }^{44}$

The motions of the molecular environment can be seen in the 2DIR spectra as changes in the shapes of the positive and negative peaks. At early waiting times, all the diagonal peaks (positive) are tilted and elongated along the diagonal line. However, at longer waiting times the peaks acquire a more up-right shape, indicating that the samples are undergoing spectral diffusion due to molecular motions. ${ }^{39}$

An interesting feature of the 2DIR spectra is the presence of cross peaks, which are offdiagonal features among the different diagonal peaks. The amide band I shows intraband cross peaks between the center amide I peak and the other two side peaks located on the low and high frequency sides $\left(\sim 1649 \mathrm{~cm}^{-1}\right.$ and $\left.\sim 1674 \mathrm{~cm}^{-1}\right)$. The waiting time evolution of these intraband amide I cross peaks not only makes these off diagonal features more visible, but also reduces the intensity of the diagonal peaks because of their spectral overlap. In addition, the amide I band presents two interband cross peaks with the amide II band $\left(\sim 1556 \mathrm{~cm}^{-1}\right)$ and with the carbonyl stretch band of the acid. While the former is clearly identifiable at longer waiting times, the latter is present even at very short waiting times. 


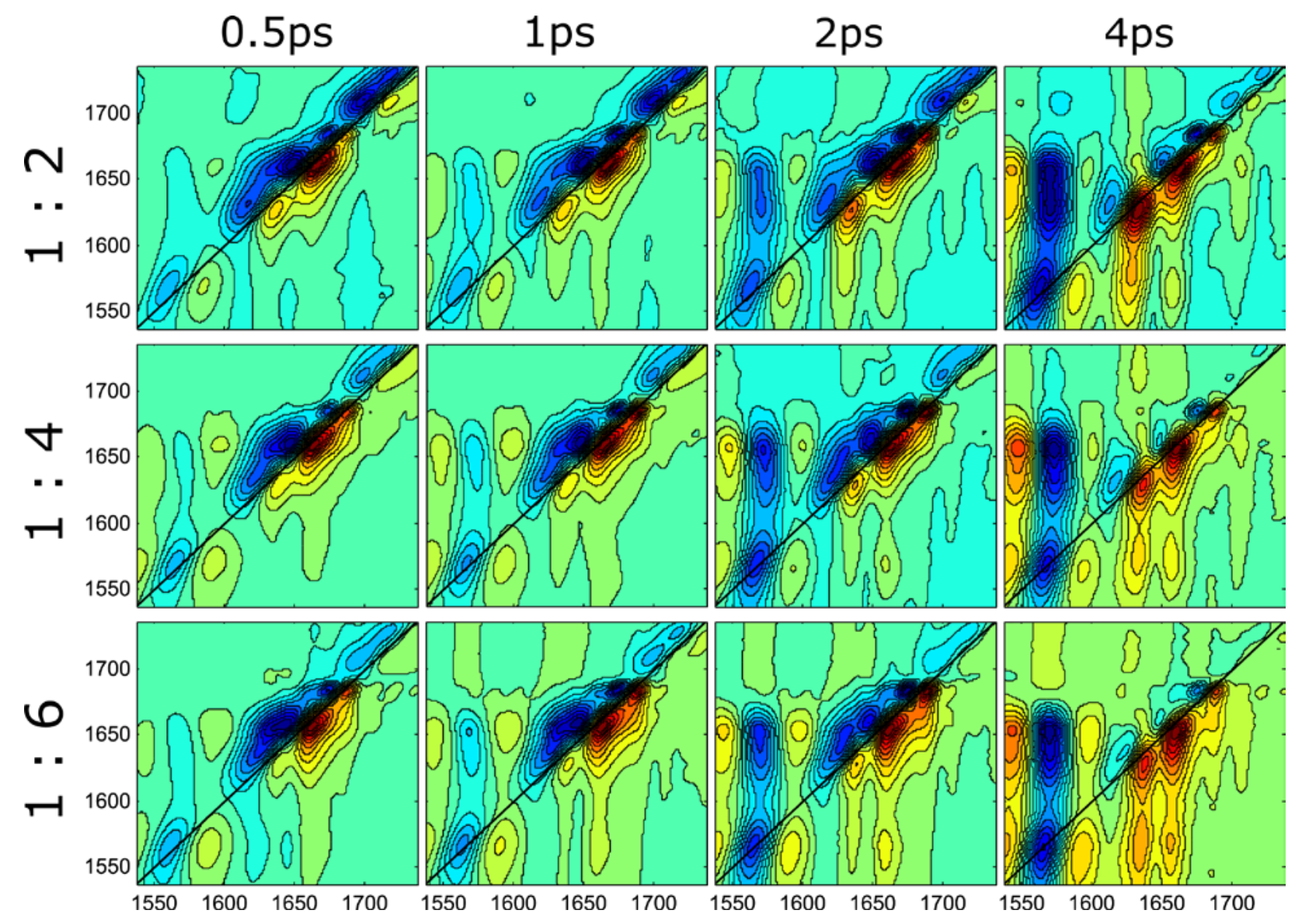

Figure 3. 2DIR spectra centered at the frequency of the amide band(s) of NMA for the different LA:NMA mixtures as described in the figure. From left to right, the waiting times are $0.5 \mathrm{ps}, 1 \mathrm{ps}$, 2 ps, and 4 ps.

The 2DIR spectroscopy in the acid bands region is presented in Figure 4. In each 2DIR spectrum, two pairs of peaks are observed in agreement with the FTIR spectra in the carbonyl region. The anharmonicity of the bands is both $\sim 16 \mathrm{~cm}^{-1}$, which agrees with the previous study. ${ }^{30}$ As shown in the previous 2DIR spectra, the diagonal peaks at $\mathrm{T}_{\mathrm{w}}=0 \mathrm{ps}$ are tilted and elongated along the diagonal line. However, these diagonal peaks do not appear to evolve within the 3 ps of investigated waiting time. In addition, the 2DIR spectra of 1:6 LA:NMA mixture presents cross peaks that are not obvious in the other two samples. For the 1:4 and 1:6 LA:NMA mixtures, an extra peak grows at $\omega_{\mathrm{t}}=\sim 1710 \mathrm{~cm}^{-1}$ at later waiting times, but it is not apparent at $\mathrm{T}_{\mathrm{w}}=0 \mathrm{ps}$. 

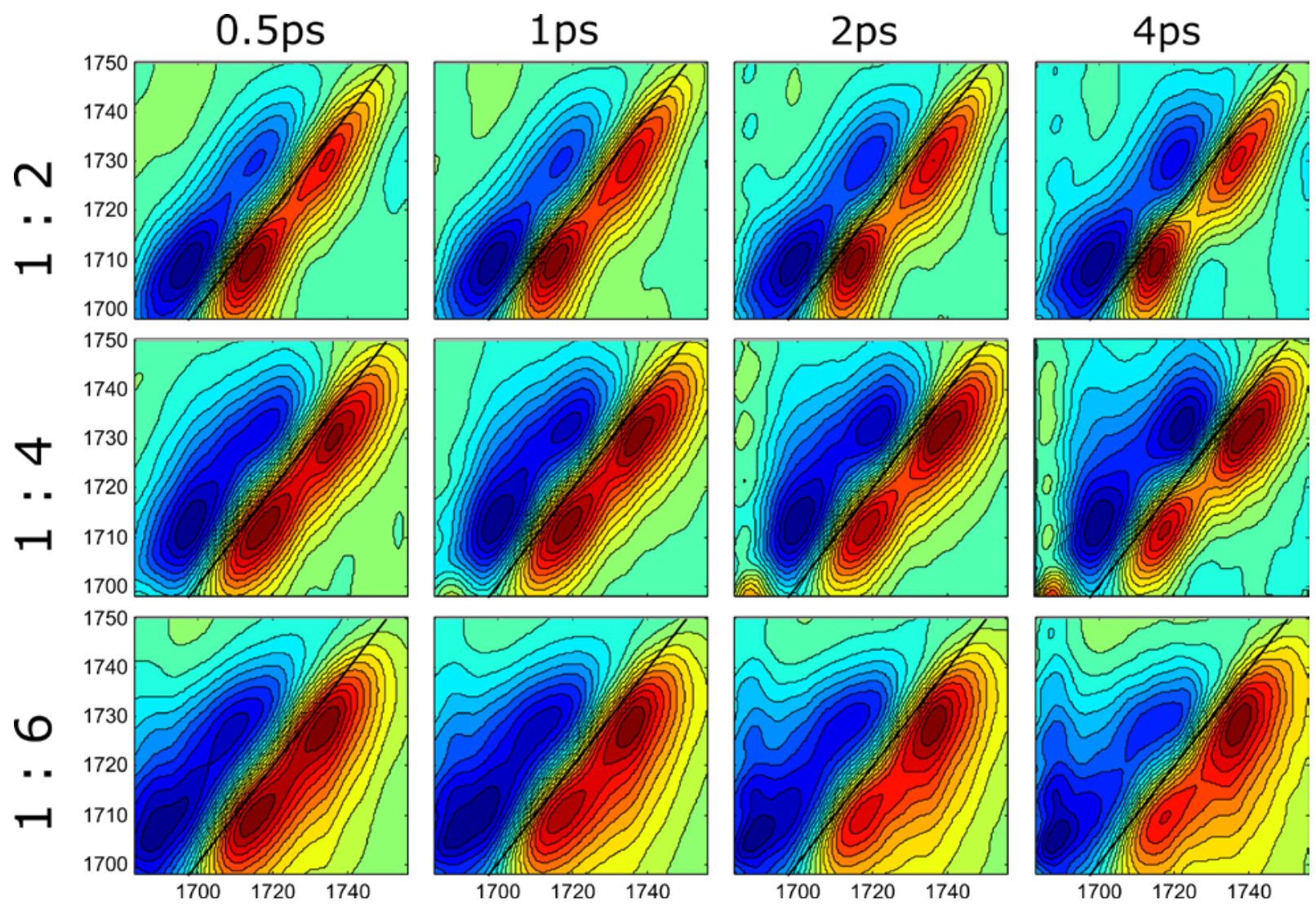

Figure 4. 2DIR spectra centered at the frequency of the carbonyl band of LA for the different LA:NMA mixtures. From left to right, the waiting times are 0.5 ps, 1 ps, 2 ps, and 4 ps.

\section{DISCUSSION}

The phase diagrams of the different mixtures of NMA-LA reveal the formation of a eutectic with a fusion point of $\sim 6{ }^{\circ} \mathrm{C}$ and a compostion in between LA:NMA molar ratio of 1:4 to 1:5. In addition, the phase diagram also shows that samples with LA:NMA molar ratios of 1:2 to 1:6 are liquid at room temperature, which allows us to investigate the structure and dynamics of the system very close to the eutectic point and outside of it.

The dynamics and interactions of the component in the mixutres were investigated via the amide I band of NMA and the carbonyl strech of LA. The amide I band of NMA has been extensively studied by experimental and theoretical methods. ${ }^{30,33,37,44-48}$ In particular, the spectra of neat NMA has been previously reported and assigned. ${ }^{44}$ The main band of the NMA amide I 
has been attributed to excitonic states of NMA where NMA molecules are hydrogen bond to one another via a single hydrogen bond between the carbonyl and the hydrogen of the amide group. The shoulder on the high-frequency side of the main band has been assigned to the non-hydrogenbonded or, equivalently, free NMA. ${ }^{44}$ In our case, two similar NMA peaks are observed, but in addition the NMA-LA samples show a low-frequency shoulder that decreases as the NMA molar ratio increases (Figure 1). This low frequency band has been previously assigned to the amide I band for amide molecules forming multiple hydrogen bonds through carbonyl oxygen. ${ }^{30,37,49}$ Thus, it is reasonable to assigned the red-side shoulder of the amide I band to NMA having two hydrogen bonds. Overall, the asymmetric profile of the FTIR spectra of DES at the amide I band region is assigned as free, one hydrogen-bonded (1HB), and two hydrogen bonded (2HB) NMA from high to low frequency.

In the spectra of NMA-LA mixtures, the two hydrogen-bonded band rises with increasing LA concentration in the mixtures, indicating that LA acts as a hydrogen bond donor, forming a hydrogen bond with NMA through its hydroxyl group. While the two hydrogen bonds could in principle be formed with other NMA or LA molecules, in pure NMA it has been shown that NMA only has one hydrogen bond through its carbonyl group ${ }^{44}$, which strongly suggests the second hydrogen bond is formed with LA molecules. Moreover, the significant difference of the amide I band profile in the DES and in NMA reveals that LA influences and modifies the hydrogen bond network of NMA. Interestingly, LA interacts with NMA, forming hydrogen bond structures in which the NMA has two hydrogen bonds, which is similar to the behaviour observed for NMA molecules in methanol. ${ }^{30}$

From the acid perspective the presence of the amide also affects the molecular interactions of LA. The carbonyl stretching modes of the acid in each LA-NMA mixture present a two-band 
profile, which contrasts with the single carbonyl peak for pure LA. The latter is consistent with the single peak observed for carboxylic acids due to intermolecular hydrogen bonding among acid molecules ${ }^{34}$ while the former shows that LA molecules can have one or two hydrogen bonds. This molecular picture of the acid in the LA-NMA mixtures also agrees with the solutions of LA in tetrahydrofuran (THF) and in methanol (see supporting information). In the case of LA in THF, a low frequency band rises at high concentrations of THF, indicating that the low frequency band of the acid corresponds to LA with a single hydrogen bond in its carbonyl group, while in LA in methanol, the number of bands and frequency positions matches those of the THF solution. Thus, the two peaks in the carbonyl region of acid peaks in the LA-NMA mixtures are assigned to LA molecules with two hydrogen-bonds (low frequency) and with one hydrogen-bond (high frequency). This assignment of the carbonyl peaks of LA in the DESs is also consistent with the linear growth $\left(\mathrm{R}^{2}=0.9950\right)$ of the area ratio between the hydrogen-bonded to LA and NMA molecules (see supporting information). In this case, an increase of the concentration of LA in the mixture, from 1:2 to 1:6, leads to the decline and growth of the LA molecules with two and one hydrogen-bond, respectively. Overall, the results show that the carbonyl groups of the acid lose hydrogen bonds with the addition of NMA, which produces not only the rise of the LA with a single hydrogen bond in its carbonyl group, but also the rise of the NMA band corresponding to two hydrogen bonds.

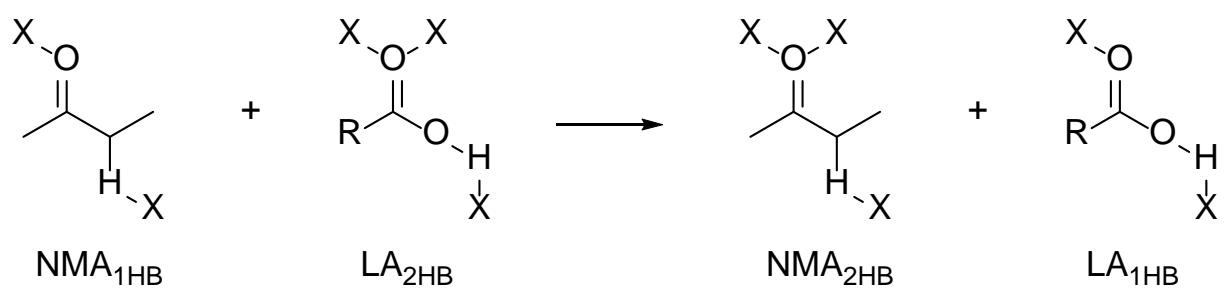

Scheme 2. Chemical equation of the DES formation. $\mathrm{X}$ represents any hydrogen bond donor molecules, such as NMA or LA. 
The thermodynamics of the hydrogen bond for the LA and NMA mixtures was extracted from the temperature-dependent FTIR spectra. ${ }^{50}$ To this end, the NMA amide I and LA carbonyl bands in all the DESs were modeled with Voight profiles for the different temperatures. The enthalpy for the formation of the DES is determined for to the chemical reaction in Scheme 2. Mathematically the enthalpy of DES formation is derived from slope of variation of the equilibrium constant $\left(K=\left(\left[N M A_{2 H B}\right]\left[L A_{1 H B}\right]\right) /\left(\left[N M A_{1 H B}\right]\left[L A_{2 H B}\right]\right)\right)$ according to Scheme 2 . The natural logarithm of the equilibrium constant as a function of the inverse temperature shows the expected linear behavior (Figure 5) and strong similarity to the slope for the different temperatures, which indicates a similar enthalpy of formation. The derived enthalpy of the DES formation shows that values might be different for the different LA:NMA molar ratios, but the error of the measurement does not allow us to draw any conclusions. However, the thermodynamics of the DES formation is not significantly affected by the altering the ratio of the components, since all the enthalpies of DES formation are within $0.5 \mathrm{~kJ} / \mathrm{mol}$ for all samples. Moreover, it is observed that the eutectic is formed even though the enthalpy of the hydrogen bond differs only $1 \mathrm{~kJ} / \mathrm{mol}$ between NMA and LA. The low magnitude of the enthalpy of DES formation shows that entropy must play a significant role in this process. 

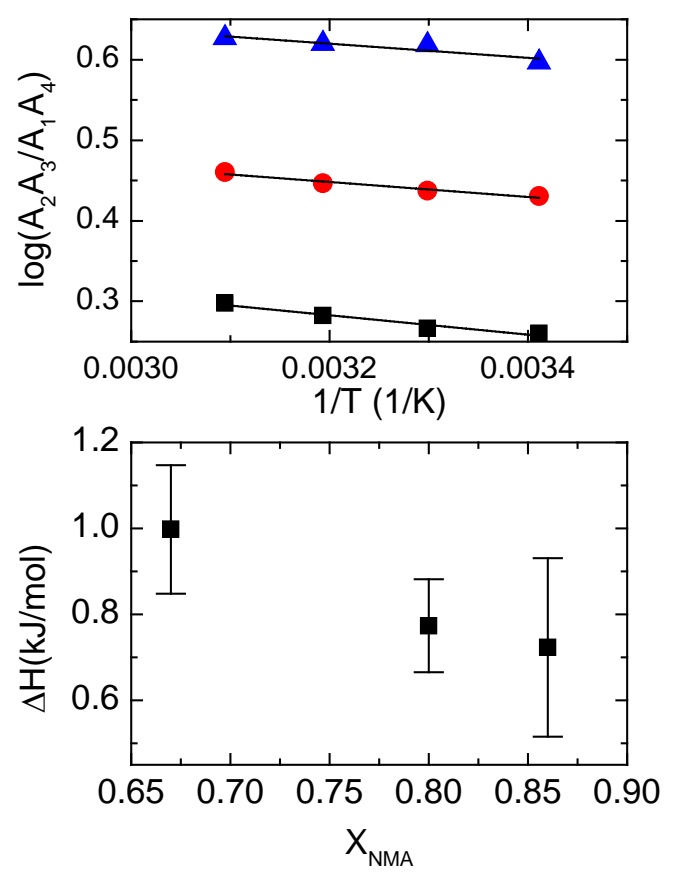

Figure 5. Thermodynamics of DES formation. The top panel depicts the natural logarithm of the ratio of areas according to Scheme 2 for 1:2 (black squares), 1:4 (red circles), and 1:6 (blue triangles) LA:NMA mixtures. The solid line indicates its linear fitting. The bottom panel shows the enthalpy change derived from the ratio of areas.

The 2DIR spectra of the LA-NMA mixtures suggests that the NMA has a similar local environment to that in neat NMA, since the amide I band in the 2DIR spectra of each mixture is similar to the 2DIR spectra of neat NMA as reported by Cunha et al. ${ }^{44}$ In other words, our results suggest that NMA molecules are very likely to see other NMA molecules even in 1:4 mixtures of LA:NMA. The observation implies a molecular picture in which the NMA molecules are forming aggregates in the liquid, and their aggregation is not affected significantly by the ratio of components. This molecular picture is consistent with formation of non-polar domains by LA due to their long carbon alkyl chains. Overall, it is likely that the LA-NMA DES has a heterogeneous structure composed of polar domains filled with NMA molecules and non-polar domain formed by the acid. In addition, the carboxylic group of LA interacts with NMA molecules in the interface that connects the polar and non-polar domains. 
The close proximity and interaction between LA and NMA can be assessed from the 2DIR spectra, since one expects that two oscillators with reasonably high transition dipole magnitudes will be vibrationally coupled if they are in close proximity. ${ }^{39}$ As a result, the 2DIR spectra should contain a cross peaks due to vibrational energy transfer. ${ }^{39}$ Indeed, the 2DIR spectra (Figure 3) shows cross peaks at $\omega_{\tau}=1600-1700 \mathrm{~cm}^{-1}$ and $\omega_{\mathrm{t}}=1710 \mathrm{~cm}^{-1}$ which are clearly visible at longer waiting times and arise from the vibrational coupling between the NMA amide I and the LA carbonyl stretch. Note that the cross peaks cannot be assigned to chemical exchange because LA cannot be converted to NMA and vice versa.

The dynamics observed by the DES components due to changes in the molecular environment was extracted from the 2DIR spectra. To this end, the CLS method was used. ${ }^{51}$ The CLS of the free amide I band in each DES is shown in Figure 6. The time evolution of the CLS shows a decay of the metric with a decay time of few picoseconds, and it is well modeled with an exponential decay of the form: $A e^{-t / \tau}+y_{0}$. The characteristic time derived from the fitting reveals a decay constant of $\sim 1-2$ ps irrespective of the sample, indicating the hydrogen bond making and breaking of the solvation shell is likely the cause of the fast dynamics in the spectral diffusion. ${ }^{30,45,52-56}$ In addition, the offset term represents slow dynamics with a characteristic time larger than the investigated window. As stated previously, the NMA-LA systems are heterogeneous at the molecular level, and so, a confinement model, similar to that of water in the reverse micelles, ${ }^{57-60}$ can be use to connect the observed CLS dynamics and the proposed heterogeneous molecular structure. Similarly, to the case of water in reverse micelle, in the DES the heterogeneous environment produces a slowdown in the dynamics of the components due to the strong effect of the interface, which propagates into the core region as the confinement is increased. ${ }^{57,61-62}$ Therefore, it is expected to observe a greater slowdown in the CLS dynamics 
when the confined volume is smaller. ${ }^{59-60,63}$ This is exactly what it is observed in the different LA:NMA samples, since all the samples show a fast dynamic component representing the core region and a slow component due to the interface. Moreover, the slow component of the amide dynamics, represented by the offset, decreases as the amount of NMA increases, which agrees with confinement since the addition of NMA increases the volume of NMA or, equivalently, reduces the confinement and consequently the slow component. Note that in this case NMA is most likely forming a continuous domain which is confined by the non-polar domains formed by the aggregation of LA molecules.

The formation of non-polar domains is further supported by the dynamics of the carbonyl groups of the acid. In this case, the CLS shows almost-constant dynamics within the investigated time window. The slow dynamics of the interface is not new and has been previously seen by others. ${ }^{57,59-60,64}$ From a molecular perspective, the carboxylate groups are most likely to be located in the interface since the carboxylic acid tail is forming the non-polar domain. Thus, the lack of CLS dynamics can be understood from the head groups being unable to move due to the interactions among tails and with the NMA molecules. Moreover, the lack of dynamics in the interfacial area is responsible for the slow dynamical component observed in the amide I band. 


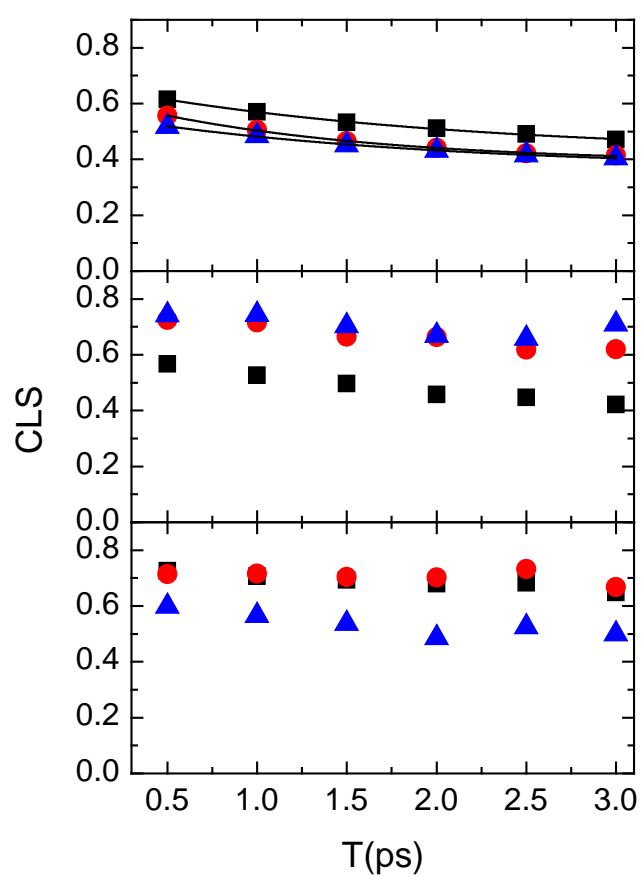

Figure 6. Spectral diffusion dynamics extracted from the 2DIR spectra. The top panel corresponds to the free amide I, and the middle and low panels to the high and low frequency carbonyl stretch bands, respectively. Black squares, red circles, and blue triangles correspond to 1:2, 1:4, and 1:6 LA:NMA mixtures, respectively. The solid black line shows the exponential fitting as described in the text.

Table 1. Parameters of exponential modeling of the CLS.

\begin{tabular}{|l|l|l|l|}
\hline LA:NMA ratio & $\mathrm{y}_{0}$ & $\mathrm{~A}$ & $\tau(\mathrm{ps})$ \\
\hline $1: 2$ & $0.42 \pm 0.01$ & $0.25 \pm 0.01$ & $1.9 \pm 0.2$ \\
\hline $1: 4$ & $0.39 \pm 0.01$ & $0.25 \pm 0.01$ & $1.3 \pm 0.1$ \\
\hline $1: 6$ & $0.36 \pm 0.01$ & $0.20 \pm 0.01$ & $1.8 \pm 0.3$ \\
\hline
\end{tabular}

The 2DIR spectra of LA:NMA mixtures also reveals that there is an overall weakening of the carboxylic acid hydrogen bond network with the addition of NMA. The decrease in the hydrogen bond strength is observed in the carbonyl stretch of the acid (Figure 4) where a cross peak is observed for 1:6 LA:NMA mixture, while the other two samples do not show any sign of this off-diagonal feature within 3 ps. The cross peak intensity between doubly and singly hydrogen bond species extracted from the 2DIR at various waiting times is shown in Figure 6. It is clear that 
the cross peak in the 1:6 LA:NMA mixture grows in intensity with time, whereas in the other samples the change is negligible. While the appearance of a cross peak related to hydrogen bond exchange is expected to be observed in 2DIR spectra of LA due to the presence of two hydrogen bond species, it is unexpected that it only appears in the sample with highest concentration of NMA. The lack of hydrogen bond exchange in the 2DIR spectra of the two samples is probably due to the higher concentration of LA, which inhibits the hydrogen bond forming and breaking process when the non-polar domain is large, probably due to the dense packing of the acid molecules at high concentrations of LA similar to that seen in bilayers and monolayers. ${ }^{65-66}$

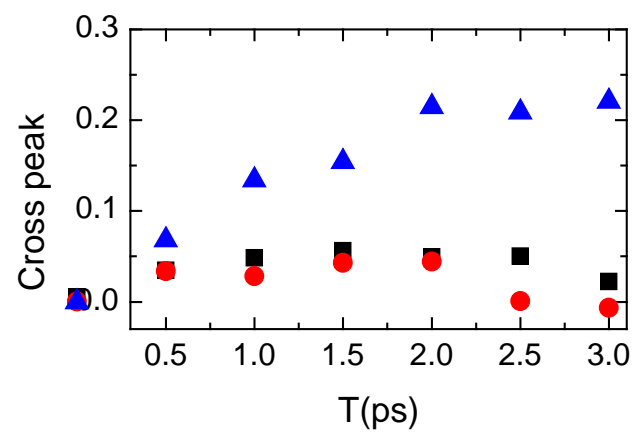

Figure 7. Cross peak growth with waiting time for DESs. Mixtures of 1:2, 1:4 and 1:6 LA:NMA are represented by black squares, red circles, and blue triangles.

The heterogeneous structure of the LA:NMA mixture was also studied via SAXS/WAXS. The SAXS patterns reveal the presence of a peak center at $0.27 \AA^{-1}$ for the samples (Figure 8), which corresponds to a real-space characteristic size of $23.3 \AA$. Interestingly, this peak position does not change significantly but has decreasing relative intensities coupled with a broader FWHM as $\mathrm{X}_{\mathrm{LA}}$ decreases. Considering the molecular size of LA, this indicates that this structural feature becomes more defined with increasing LA micelle formation, suggesting that the LA is key to forming structural order in the DES. The WAXS patterns show another peak that shifts to higher q with decreasing $X_{\text {LA }}$ (Figure 8). At a 2:1 NMA:LA ratio, a WAXS peak is found at $1.43 \AA^{-1}$, corresponding to a d-spacing of $4.39 \AA$. For the lower $\mathrm{X}_{\mathrm{LA}} \mathrm{DES}$ samples, this peak is located at 
$1.50 \AA^{-1}$, which corresponds to a d-spacing of $4.19 \AA$. Note that his peak is more anisotropic in these two DES mixtures, suggesting that heterogenous ordering at this length scale. Given the size associated with these peaks, it is likely that this feature corresponds to local ordering of the NMA through the proposed hydrogen-bonding mechanisms as described above. Notably, this ordering is directly depended on LA:NMA ratio. Moreover, the SAXS/WAXS experiments serves to directly support the heterogeneous structure of the NMA-LA DES.

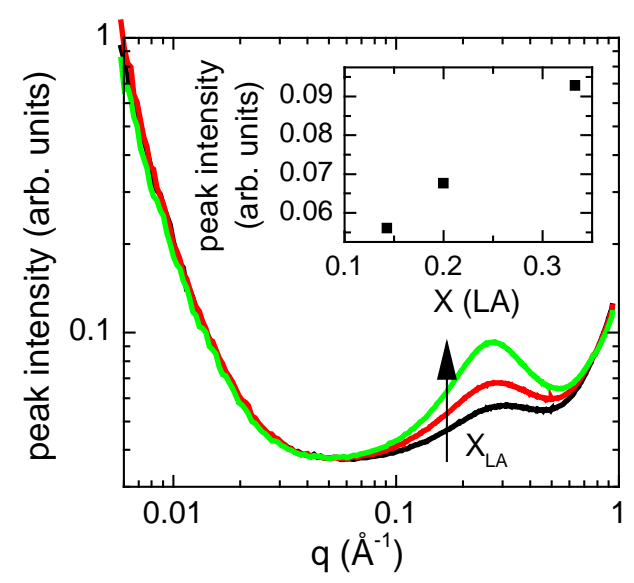

Figure 8. Small angle X-ray scattering of the NMA-LA mixtures. Green, red, and black lines represent the mixtures with decreasing amount of LA. The inset shows the intensity of the SAXS peak at $\sim 0.28 \AA^{-1}$ as a function of the molar fraction of LA.

\section{SUMMARY}

The interactions and dynamics of NMA and LA in the DESs were investigated by linear and time-resolved infrared spectroscopy. This study shows that NMA and LA interact via hydrogen bonding. In the DES, the carbonyl group of NMA is shown to form none, one, or two hydrogen-bonds, while and the carbonyl of the acid is found with either two or one hydrogenbond. Moreover, the stabilization energy for the formation of the liquid is found to be $\sim 1 \mathrm{~kJ} / \mathrm{mol}$. Interesting, in LA-NMA mixtures of similar molar ratio, the interactions between the molecules of the same kind remained. On the other hand, the dynamics of the environment derived from the 
time resolved experiments shows a fast and a slow components for NMA and only a slow component for the acid. Based on the interactions between components and the dynamics probed by NMA amide I band and the LA carbonyl group, it is proposed that the NMA-LA system has a molecular heterogeneous structure with polar and non-polar domains. In this molecular picture, the amide forms a continuum with segregated non-polar domains. The proposed heterogeneous structure of the NMA-LA DES systems is directly verified by small angle X-ray scattering. The SAXS/WAXS data shows that the LA-NMA mixtures have nanoscopic heterogeneities that are dependent on the composition of the mixture.

\section{ASSOCIATED CONTENT}

\section{Supporting Information}

Figures S1 to S3, FTIR spectra of LA in THF, FTIR spectrum of LA in methanol, and the ratio of the areas as of the one and two hydrogen bond species of LA as function of the molar fraction of LA. This information is available free of charge via the Internet at http://pubs.acs.org.

\section{AUTHOR INFORMATION}

\section{Corresponding Author}

* E-mail: dkuroda@lsu.edu. Tel: (+1) 225-578-1780

\section{ORCID}

Daniel G. Kuroda: 0000-0002-4752-7024

\section{NOTES}


The authors declare no competing financial interest.

\section{ACKNOWLEDGMENTS}

The current work was supported in part by start-up funds provided to DGK by the LSU Department of Chemistry and by the ACS - Petroleum Research Fund grant 56529-DNI6. This research used the 12-ID-B beamline of the Advanced Photon Source, a U.S. Department of Energy (DOE) Office of Science User Facility operated for the DOE Office of Science by Argonne National Laboratory under Contract No. DE-AC02-06CH11357.

\section{REFERENCES}

1. $\quad$ Guthrie, F., On Eutexia. Proc. Phys. Soc. 1884, 6, 124.

2. Abbott, A. P.; Barron, J. C.; Ryder, K. S.; Wilson, D., Eutectic - Based Ionic Liquids with Metal - Containing Anions and Cations. Chem. Eur. J. 2007, 13, 6495-6501.

3. $\quad$ Abbott, A. P.; Capper, G.; Davies, D. L.; Munro, H. L.; Rasheed, R. K.; Tambyrajah, V., Preparation of Novel, Moisture-Stable, Lewis-Acidic Ionic Liquids Containing Quaternary Ammonium Salts with Functional Side Chains. Chem. Commun. 2001, 2010-2011.

4. Abbott, A. P.; Boothby, D.; Capper, G.; Davies, D. L.; Rasheed, R. K., Deep Eutectic Solvents Formed between Choline Chloride and Carboxylic Acids: Versatile Alternatives to Ionic Liquids. J. Am. Chem. Soc. 2004, 126, 9142-9147.

5. Smith, E. L.; Abbott, A. P.; Ryder, K. S., Deep Eutectic Solvents (Dess) and Their Applications. Chem. Rev. 2014, 114, 11060-11082.

6. Zhang, Q.; Vigier, K. D. O.; Royer, S.; Jerome, F., Deep Eutectic Solvents: Syntheses, Properties and Applications. Chem. Soc. Rev. 2012, 41, 7108-7146.

7. Zhao, H.; Baker, G. A., Ionic Liquids and Deep Eutectic Solvents for Biodiesel Synthesis: A Review. J. Chem. Technol. Biotechnol. 2013, 88, 3-12.

8. Francisco, M.; van den Bruinhorst, A.; Kroon, M. C., Low - Transition - Temperature Mixtures (Lttms): A New Generation of Designer Solvents. Angew. Chem. Int. Ed. 2013, 52, 30743085.

9. Welton, T., Room-Temperature Ionic Liquids. Solvents for Synthesis and Catalysis. Chem. Rev. 1999, 99, 2071-2084.

10. Garcia, G.; Aparicio, S.; Ullah, R.; Atilhan, M., Deep Eutectic Solvents: Physicochemical Properties and Gas Separation Applications. Energy Fuels 2015, 29, 2616-2644.

11. Singh, B.; Lobo, H.; Shankarling, G., Selective N-Alkylation of Aromatic Primary Amines Catalyzed by Bio-Catalyst or Deep Eutectic Solvent. Catal. Lett. 2011, 141, 178-182.

12. Yang, H.; Guo, X.; Birbilis, N.; Wu, G.; Ding, W., Tailoring Nickel Coatings Via Electrodeposition from a Eutectic-Based Ionic Liquid Doped with Nicotinic Acid. Appl. Surf. Sci. 2011, 257, 9094-9102. 
13. Gómez, E.; Cojocaru, P.; Magagnin, L.; Valles, E., Electrodeposition of Co, Sm and Smco from a Deep Eutectic Solvent. J. Electroanal. Chem. 2011, 658, 18-24.

14. Abbott, A. P.; Capper, G.; Davies, D. L.; McKenzie, K. J.; Obi, S. U., Solubility of Metal Oxides in Deep Eutectic Solvents Based on Choline Chloride. J. Chem. Eng. Data 2006, 51, 12801282.

15. Leron, R. B.; Li, M.-H., Solubility of Carbon Dioxide in a Choline Chloride-Ethylene Glycol Based Deep Eutectic Solvent. Thermochim. Acta 2013, 551, 14-19.

16. Shahbaz, K.; Mjalli, F.; Hashim, M.; AlNashef, I., Using Deep Eutectic Solvents Based on Methyl Triphenyl Phosphunium Bromide for the Removal of Glycerol from Palm-Oil-Based Biodiesel. Energy Fuels 2011, 25, 2671-2678.

17. Carriazo, D.; Serrano, M. C.; Gutiérrez, M. C.; Ferrer, M. L.; del Monte, F., Deep-Eutectic Solvents Playing Multiple Roles in the Synthesis of Polymers and Related Materials. Chem. Soc. Rev. 2012, 41, 4996-5014.

18. Fortenberry, D. I.; Pojman, J. A., Solvent - Free Synthesis of Polyacrylamide by Frontal Polymerization. J. Polym. Sci., Part A: Polym. Chem. 2000, 38, 1129-1135.

19. Cui, Y.; Li, M.-C.; Wu, Q.; Pojman, J. A.; Kuroda, D. G., Synthesis-Free Phase-Selective Gelator for Oil-Spill Remediation. ACS applied materials and interfaces 2017, 9, 33549-33553.

20. Cui, Y.; Fulfer, K.; Ma, J.; Weldeghiorghis, T.; Kuroda, D., Solvation Dynamics of an Ionic Probe in Choline Chloride-Based Deep Eutectic Solvents. PCCP 2016, 18, 31471-31479.

21. Perkins, S. L.; Painter, P.; Colina, C. M., Experimental and Computational Studies of Choline Chloride-Based Deep Eutectic Solvents. J. Chem. Eng. Data 2014, 59, 3652-3662.

22. Das, A.; Biswas, R., Dynamic Solvent Control of a Reaction in Ionic Deep Eutectic Solvents: Time-Resolved Fluorescence Measurements of Reactive and Nonreactive Dynamics in (Choline Chloride+ Urea) Melts. J. Phys. Chem. B 2015, 119, 10102-10113.

23. Wagle, D. V.; Baker, G. A.; Mamontov, E., Differential Microscopic Mobility of Components within a Deep Eutectic Solvent. Journal of physical chemistry letters 2015, 6, 29242928.

24. D'Agostino, C.; Harris, R. C.; Abbott, A. P.; Gladden, L. F.; Mantle, M. D., Molecular Motion and Ion Diffusion in Choline Chloride Based Deep Eutectic Solvents Studied by $1 \mathrm{H}$ Pulsed Field Gradient Nmr Spectroscopy. PCCP 2011, 13, 21383-21391.

25. Cui, Y. W.; Kuroda, D. G., Evidence of Molecular Heterogeneities in Amide-Based Deep Eutectic Solvents. J. Phys. Chem. A 2018, 122, 1185-1193.

26. Pandey, A.; Rai, R.; Pal, M.; Pandey, S., How Polar Are Choline Chloride-Based Deep Eutectic Solvents? PCCP 2014, 16, 1559-1568.

27. Makos, P.; Przyjazny, A.; Boczkaj, G., Hydrophobic Deep Eutectic Solvents as "Green" Extraction Media for Polycyclic Aromatic Hydrocarbons in Aqueous Samples. J. Chromatogr. A 2018, 1570, 28-37.

28. van Osch, D. J.; Zubeir, L. F.; van den Bruinhorst, A.; Rocha, M. A.; Kroon, M. C., Hydrophobic Deep Eutectic Solvents as Water-Immiscible Extractants. Green Chem. 2015, 17, 4518-4521.

29. Das, A.; Das, S.; Biswas, R., Density Relaxation and Particle Motion Characteristics in a Non-Ionic Deep Eutectic Solvent (Acetamide+ Urea): Time-Resolved Fluorescence Measurements and All-Atom Molecular Dynamics Simulations. J. Chem. Phys. 2015, 142, 034505.

30. Woutersen, S.; Mu, Y.; Stock, G.; Hamm, P., Hydrogen-Bond Lifetime Measured by TimeResolved 2d-Ir Spectroscopy: N-Methylacetamide in Methanol. Chem. Phys. 2001, 266, 137-147. 
31. Asplund, M.; Zanni, M. T.; Hochstrasser, R. M., Two-Dimensional Infrared Spectroscopy of Peptides by Phase-Controlled Femtosecond Vibrational Photon Echoes. PNAS 2000, 97, 82198224.

32. DeFlores, L. P.; Ganim, Z.; Nicodemus, R. A.; Tokmakoff, A., Amide I' - Ii $^{\prime}$ 2d Ir Spectroscopy Provides Enhanced Protein Secondary Structural Sensitivity. J. Am. Chem. Soc. 2009, 131, 3385-3391.

33. DeCamp, M.; DeFlores, L.; McCracken, J.; Tokmakoff, A.; Kwac, K.; Cho, M., Amide I Vibrational Dynamics of N-Methylacetamide in Polar Solvents: The Role of Electrostatic Interactions. J. Phys. Chem. B 2005, 109, 11016-11026.

34. Lim, M.; Hochstrasser, R. M., Unusual Vibrational Dynamics of the Acetic Acid Dimer. J. Chem. Phys. 2001, 115, 7629-7643.

35. Rubtsov, I. V.; Wang, J.; Hochstrasser, R. M., Dual-Frequency 2d-Ir Spectroscopy Heterodyned Photon Echo of the Peptide Bond. PNAS 2003, 100, 5601-5606.

36. Kim, Y. S.; Hochstrasser, R. M., Applications of 2d Ir Spectroscopy to Peptides, Proteins, and Hydrogen-Bond Dynamics. J. Phys. Chem. B 2009, 113, 8231-8251.

37. Ganim, Z.; Chung, H. S.; Smith, A. W.; DeFlores, L. P.; Jones, K. C.; Tokmakoff, A., Amide I Two-Dimensional Infrared Spectroscopy of Proteins. Acc. Chem. Res. 2008, 41, 432-441.

38. Wang, L.; Middleton, C. T.; Zanni, M. T.; Skinner, J. L., Development and Validation of Transferable Amide I Vibrational Frequency Maps for Peptides. J. Phys. Chem. B 2011, 115, 37133724.

39. Hamm, P.; Zanni, M., Concepts and Methods of 2d Infrared Spectroscopy; Cambridge University Press, 2011.

40. Fayer, M., Dynamics of Liquids, Molecules, and Proteins Measured with Ultrafast 2d Ir Vibrational Echo Chemical Exchange Spectroscopy. Annu. Rev. Phys. Chem. 2009, 60, 21-38.

41. Kim, Y. S.; Wang, J.; Hochstrasser, R. M., Two-Dimensional Infrared Spectroscopy of the Alanine Dipeptide in Aqueous Solution. J. Phys. Chem. B 2005, 109, 7511-7521.

42. Fulfer, K.; Kuroda, D., A Comparison of the Solvation Structure and Dynamics of the Lithium Ion in Linear Organic Carbonates with Different Alkyl Chain Lengths. PCCP 2017, 19, 25140-25150.

43. Besley, N. A., Ab Initio Modeling of Amide Vibrational Bands in Solution. J. Phys. Chem. A 2004, 108, 10794-10800.

44. Cunha, A. V.; Salamatova, E.; Bloem, R.; Roeters, S. J.; Woutersen, S.; Pshenichnikov, M. S.; Jansen, T. L., Interplay between Hydrogen Bonding and Vibrational Coupling in Liquid NMethylacetamide. journal of physical chemistry letters 2017, 8, 2438-2444.

45. Zanni, M. T.; Asplund, M. C.; Hochstrasser, R. M., Two-Dimensional Heterodyned and Stimulated Infrared Photon Echoes of N-Methylacetamide-D. J. Chem. Phys. 2001, 114, 45794590 .

46. Kwac, K.; Cho, M., Molecular Dynamics Simulation Study of N-Methylacetamide in Water. Ii. Two-Dimensional Infrared Pump-Probe Spectra. J. Chem. Phys. 2003, 119, 2256-2263. 47. DeFlores, L. P.; Ganim, Z.; Ackley, S. F.; Chung, H. S.; Tokmakoff, A., The Anharmonic Vibrational Potential and Relaxation Pathways of the Amide I and Ii Modes of NMethylacetamide. J. Phys. Chem. B 2006, 110, 18973-18980.

48. Torii, H.; Tatsumi, T.; Tasumi, M., Effects of Hydration on the Structure, Vibrational Wavenumbers, Vibrational Force Field and Resonance Raman Intensities of $\mathrm{N}$ Methylacetamide. J. Raman Spectrosc. 1998, 29, 537-546. 
49. Kwac, K.; Lee, H.; Cho, M., Non-Gaussian Statistics of Amide I Mode Frequency Fluctuation of N-Methylacetamide in Methanol Solution: Linear and Nonlinear Vibrational Spectra. J. Chem. Phys. 2004, 120, 1477-1490.

50. Guerin, A. C.; Riley, K.; Rupnik, K.; Kuroda, D. G., Determining the Energetics of the Hydrogen Bond through Ftir: A Hands-on Physical Chemistry Lab Experiment. J. Chem. Educ. 2016, 93, 1124-1129.

51. Fenn, E. E.; Fayer, M. D., Extracting 2d Ir Frequency-Frequency Correlation Functions from Two Component Systems. J. Chem. Phys. 2011, 135, 074502.

52. Fecko, C. J.; Eaves, J. D.; Loparo, J. J.; Tokmakoff, A.; Geissler, P. L., Ultrafast HydrogenBond Dynamics in the Infrared Spectroscopy of Water. Science 2003, 301, 1698-1702.

53. Woutersen, S.; Pfister, R.; Hamm, P.; Mu, Y.; Kosov, D. S.; Stock, G., Peptide Conformational Heterogeneity Revealed from Nonlinear Vibrational Spectroscopy and MolecularDynamics Simulations. J. Chem. Phys. 2002, 117, 6833-6840.

54. Ghosh, A.; Hochstrasser, R. M., A Peptide’s Perspective of Water Dynamics. Chem. Phys. 2011, 390, 1-13.

55. Chung, J. K.; Thielges, M. C.; Fayer, M. D., Dynamics of the Folded and Unfolded Villin Headpiece (Hp35) Measured with Ultrafast 2d Ir Vibrational Echo Spectroscopy. PNAS 2011, 108, 3578-3583.

56. Ghosh, A.; Remorino, A.; Tucker, M. J.; Hochstrasser, R. M., 2d Ir Photon Echo Spectroscopy Reveals Hydrogen Bond Dynamics of Aromatic Nitriles. Chem. Phys. Lett. 2009, 469, 325-330.

57. Singh, P. K.; Kuroda, D. G.; Hochstrasser, R. M., An Ion's Perspective on the Molecular Motions of Nanoconfined Water: A Two-Dimensional Infrared Spectroscopy Study. J. Phys. Chem. B 2013, 117, 9775-9784.

58. Tan, H.-S.; Piletic, I. R.; Riter, R. E.; Levinger, N. E.; Fayer, M., Dynamics of Water Confined on a Nanometer Length Scale in Reverse Micelles: Ultrafast Infrared Vibrational Echo Spectroscopy. Phys. Rev. Lett. 2005, 94, 057405.

59. Moilanen, D. E.; Fenn, E. E.; Wong, D.; Fayer, M. D., Water Dynamics in Large and Small Reverse Micelles: From Two Ensembles to Collective Behavior. J. Chem. Phys. 2009, 131, 014704.

60. Moilanen, D. E.; Fenn, E. E.; Wong, D.; Fayer, M., Water Dynamics at the Interface in Aot Reverse Micelles. J. Phys. Chem. B 2009, 113, 8560-8568.

61. Yuan, R. F.; Yan, C.; Nishida, J.; Fayer, M. D., Dynamics in a Water Interfacial Boundary Layer Investigated with Ir Polarization-Selective Pump-Probe Experiments. J. Phys. Chem. B 2017, 121, 4530-4537.

62. Roy, V. P.; Kubarych, K. J., Interfacial Hydration Dynamics in Cationic Micelles Using 2d-Ir and Nmr. J. Phys. Chem. B 2017, 121, 9621-9630.

63. Piletic, I. R.; Moilanen, D. E.; Spry, D. B.; Levinger, N. E.; Fayer, M. D., Testing the Core/Shell Model of Nanoconfined Water in Reverse Micelles Using Linear and Nonlinear Ir Spectroscopy. J. Phys. Chem. A 2006, 110, 4985-4999.

64. Lee, J.; Maj, M.; Kwak, K.; Cho, M., Infrared Pump-Probe Study of Nanoconfined Water Structure in Reverse Micelle. journal of physical chemistry letters 2014, 5, 3404-3407.

65. Yan, C.; Thomaz, J. E.; Wang, Y. L.; Nishida, J.; Yuan, R. F.; Breen, J. P.; Fayer, M. D., Ultrafast to Ultraslow Dynamics of a Langmuir Monolayer at the Air/Water Interface Observed with Reflection Enhanced 2d Ir Spectroscopy. J. Am. Chem. Soc. 2017, 139, 16518-16527. 
66. Volkov, V. V.; Chelli, R.; Righini, R., Domain Formation in Lipid Bilayers Probed by Two-Dimensional Infrared Spectroscopy. J. Phys. Chem. B 2006, 110, 1499-1501. 


\section{Supporting information}

\section{The Molecularly Heterogeneous Structure of Non- Ionic Deep Eutectic Solvent Composed of N- Methylacetamide and Lauric Acid}

Yaowen Cui,† Jeramie Rushing, † Soenke Seifert, $\ddagger$ Nicholas M. Bedford,§ and Daniel G. Kuroda†*

†Department of Chemistry, Louisiana State University, Baton Rouge, Louisiana 70803, United

States

$\ddagger X$-ray Sciences Division, Argonne National Laboratory, Argonne, IL, 60439, USA

§School of Chemical Engineering, University of New South Wales, Sydney, NSW, 2052, AUS

*Address correspondence to dkuroda@lsu.edu 


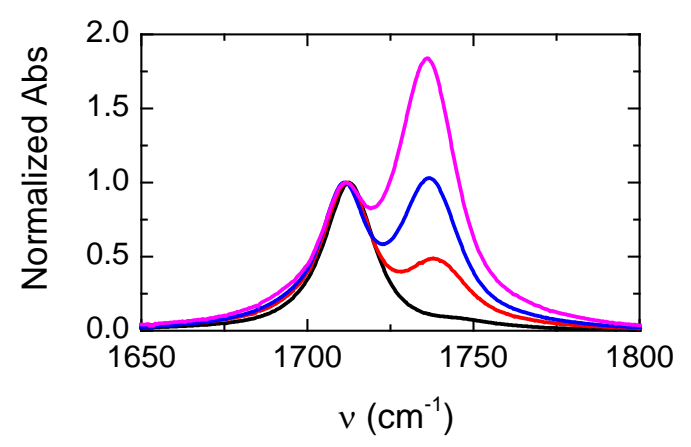

Figure S1. FTIR spectra of melted LA and LA in THF with different concentrations. The spectra are normalized according to the low-frequency band.

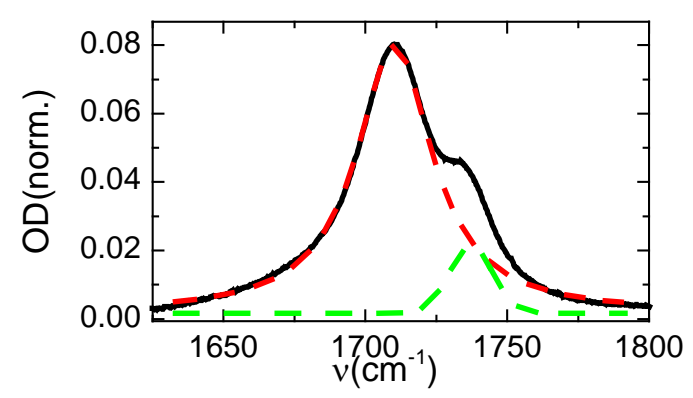

Figure S2. FTIR spectra of LA in methanol at $50 \mathrm{mM}$. The carbonyl stretches of LA are fitted with a two-band voigt function. The frequencies of the two bands are $1710 \mathrm{~cm}^{-1}$ and $1737 \mathrm{~cm}^{-1}$.

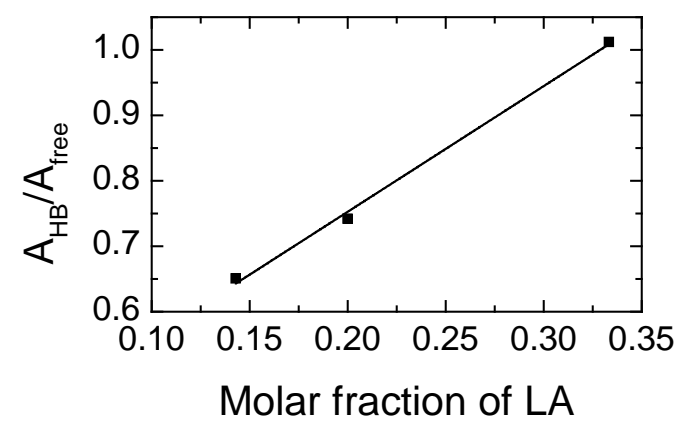

Figure S3. Area ratios of the two hydrogen-bond band over one hydrogen-bond band of LA carbonyl stretch for various LA:NMA molar ratios. The solid line is the linear fitting. 